\title{
Expression and Localization of the Cell Adhesion Molecule SgIGSF during Regeneration of the Olfactory Epithelium in Mice
}

\author{
Fusae Tsukioka ${ }^{1,2}$, Tomohiko Wakayama ${ }^{1}$, Toshiaki Tsukatani², Takaki Miwa², \\ Mitsuru Furukawa² ${ }^{2}$ and Shoichi Iseki ${ }^{1}$ \\ ${ }^{\prime}$ Department of Histology and Embryology, Graduate School of Medical Science, Kanazawa University, Kanazawa, Japan and \\ ${ }^{2}$ Department of Otorhinolaryngology, Graduate School of Medical Science, Kanazawa University, Kanazawa, Japan
}

Received December 15, 2006; accepted February 20, 2007; published online April 6, 2007

\begin{abstract}
Spermatogenic immunoglobulin superfamily (SgIGSF) is a cell adhesion molecule originally discovered in mouse testis. SgIGSF is expressed not only in spermatogenic cells but also in lung and liver epithelial cells and in neurons and glia of the central and peripheral nervous systems. In the present study, we examined the expression and localization of SgIGSF in mouse olfactory epithelium before and after transection of the olfactory nerves, by RT-PCR, Western blotting and immunohistochemistry. In normal olfactory mucosa, SgIGSF showed $100 \mathrm{kDa}$ in molecular weight, which was identical with that in the lung but different from that in the brain. SgIGSF was expressed on the membrane of all olfactory, sustentacular and basal cells, but more abundantly in the apical portions of the olfactory epithelium where the dendrites of olfactory cells are in contact with sustentacular cells. After olfactory nerve transection, mature olfactory cells disappeared in 4 days but were regenerated around 715 days by proliferation and differentiation of basal cells into mature olfactory cells through the step of immature olfactory cells. During this period, both the mRNA and protein for SgIGSF showed a transient increase, with peak levels at 7 days and 11 days, respectively, after the transection. Immunohistochemistry showed that the enriched immunoreactivity for SgIGSF at 7-11 days was localized primarily to the membrane of immature olfactory cells. These results suggested that, during regeneration of the olfactory epithelium, the adhesion molecule SgIGSF plays physiological roles in differentiation, migration, and maturation of immature olfactory cells.
\end{abstract}

Key words: cell adhesion molecule, SgIGSF, olfactory epithelium, regeneration

\section{Introduction}

The olfactory epithelium is composed of olfactory cells, sustentacular cells and basal cells [6]. The olfactory cell is unique in that it functions as the primary sensory neuron while performing continuous degeneration and regeneration throughout the animal life. The apical end of the dendrites of olfactory cells forms the olfactory vesicle, from which olfactory cilia extend toward the nasal cavity. The basal portion of olfactory cells extends a long unmyelinated

Correspondence to: Shoichi Iseki, M.D., Ph.D., Department of Histology and Embryology, Graduate School of Medical Science, Kanazawa University, 13-1 Takara-machi, Kanazawa 920-8640, Japan.

E-mail: siseki@med.kanazawa-u.ac.jp axon through the lamina propria. The axons from different olfactory cells form many bundles called olfactory nerves (filaments) that reach the olfactory bulb, where they synapse with the pyramidal cells of the secondary olfactory neuron. The transection of olfactory nerves in mice was first reported by Graziadei and Graziadei [7]. This method enables selective resection of all olfactory axons without injuring the olfactory cell body or olfactory bulb. After transection, the distal stumps of the olfactory nerves undergo Wallerian degeneration, followed by apoptosis in all olfactory cells, resulting in extensive atrophy of the olfactory epithelium. Subsequently, the basal cells undergo extensive proliferation, differentiation, and maturation to form new olfactory cells, giving rise to the complete regeneration of the olfactory epithelium. Therefore, this system of olfactory nerve 
transection makes it possible to reproduce the process of olfactory neuron turnover in a synchronized manner.

The olfactory cell, despite its being a bipolar neuron, forms junctional complexes composed of tight junctions, adherence junctions, and desmosomes with neighboring sustentacular cells or basal cells. Therefore, it seems reasonable to assume that cell adhesion molecules play certain roles in the functional integrity of olfactory cells. As an adhesion molecule expressed in the olfactory epithelium, the neural cell adhesion molecule (NCAM) is well documented [16]. NCAM is expressed abundantly throughout the nervous system. In the olfactory epithelium, NCAM is localized to the membrane of both immature and mature olfactory cells and their axons [26]. During development of the nervous system, NCAM is considered to play an important role in neural migration, nerve fiber elongation, nerve bundle formation, as well as synaptic formation and plasticity [15]. Recently, it was reported that a transient increase in the expression of NCAM occurs in neurons after transection of the sciatic nerve, suggesting that NCAM is also involved in regeneration of the peripheral nervous system [20]. In the olfactory epithelium, there is one report that the level of NCAM expression is elevated transiently after resection of the olfactory bulb [29].

The adhesion molecule spermatogenic immunoglobulin superfamily (SgIGSF) was originally reported in 2001 by Wakayama et al. [23] as a molecule expressed on the membrane of spermatogenic cells in the mouse testis. Like NCAM, SgIGSF belongs to the immunoglobulin superfamily (IGSF) adhesion molecules, which are characterized by having extracellular regions containing Ig-like domains [3]. In the testis, SgIGSF is considered to be involved in the adhesion between spermatogenic and Sertoli cells, thereby playing roles in spermatogenesis [24]. A mouse strain lacking the SgIGSF gene is known to have male infertility due to a remarkable decrease in the number of mature spermatozoa [28]. Immunohistochemical studies in adult mice demonstrated that SgIGSF is expressed not only by spermatogenic cells but also by hepatocytes and bile duct epithelial cells in the liver, alveolar epithelial cells in the lung, mast cells in the connective tissue, as well as neurons and glias in the central and peripheral nervous system [9, 24, 25]. SgIGSF and its human homologue have also been reported in the names of IgSF4, TSLC1, RA175, SynCAM, and Necl-2 [2, $5,10,18,21]$. In a cultured cell system, an aberrant synaptic formation occurs between neurons and the non-neuronal cells forced to express SynCAM, suggesting that SgIGSF is involved in neuronal plasticity [2]. In developing mice, SgIGSF was shown to be distributed diffusely in the central nervous system and sensory epithelia, including the olfactory epithelium at embryonic day 16.5 [4].

In the present study, we aimed to examine the expression and localization of SgIGSF in the normal adult mouse olfactory epithelium and in the epithelium undergoing regeneration after olfactory nerve transection.

\section{Materials and Methods}

\section{Animals and transection of the olfactory nerve}

Male ICR mice at 8 weeks of age were purchased from Nippon SLC, Inc. (Hamamatsu, Japan) and grown under standard laboratory conditions with a $12 \mathrm{hr}$-light/12 hr-dark cycle and free access to food and water. All animal experiments were performed according to the Guidelines for the Care and Use of Laboratory Animals in Kanazawa University. Under an anesthesia with intraperitoneal injection of pentobarbital at a concentration of $60 \mathrm{mg} / \mathrm{kg}$, the animals underwent transection of the olfactory nerves according to the method described previously [7, 30]. Briefly, a small hole was made in the frontal bone of the skull using a microdrill and, using a teflon knife inserted into this hole, the olfactory nerves on both sides were severed between the Lamina cribrosa of ethmoid bone and the olfactory bulbs. We confirmed this procedure causes loss of the sense of smell in mice as judged by behavior analysis. Thereafter, the animals were raised for 4 to 35 days under standard laboratory conditions at $22^{\circ} \mathrm{C}$ with a $12 \mathrm{hr}$-light $/ 12 \mathrm{hr}$-dark cycle and free access to food and water. At 0 day (control) and 4, $7,11,15$ and 35 days after olfactory nerve transection, the animals were sacrificed with an intraperitoneal injection of pentobarbital. Three to 4 animals were used for each time point, and the whole experiment was repeated 3 times. For RT-PCR and Western blot analyses, the portion of the skull covering the nasal cavity of two animals was removed, and the olfactory mucosa on both sides of the nasal cavity were separately exfoliated with forceps, frozen immediately in a liquid nitrogen bath and stored at $-80^{\circ} \mathrm{C}$ prior to use. For immunohistochemistry, another 1 to 2 animals were fixed by transcardial perfusion with physiological saline followed by $4 \%$ paraformaldehyde in $0.1 \mathrm{M}$ phosphate buffer ( $\mathrm{pH}$ 7.2). Animals were decapitated, the head cleared of skin and muscles, further fixed by immersion in the same fixative for $4 \mathrm{hr}$, and then dipped in $0.5 \mathrm{M}$ EDTA for 5 days at $4{ }^{\circ} \mathrm{C}$ for decalcification of bone. Decalcified specimens were either frozen in liquid nitrogen for immunoelectron microscopy, or dehydrated in ethanol series and embedded in paraffin for light microscopic immunohistochemistry.

\section{$R N A$ preparation and reverse transcription-polymerase chain reaction (RT-PCR)}

Total RNA was isolated from the frozen specimens by disrupting them in a guanidinine-based solution (TRI reagent; Sigma-Aldrich Co., St. Louis, MO) followed by purifying RNA using RNeasy Mini Kit (Quiagen $\mathrm{GmbH}$, Hilden, Germany) in accordance with the manufacturer's instructions. First-strand cDNA was synthesized from $2 \mu \mathrm{g}-$ aliquot of total RNA samples using the oligo(dT)20 primer and Moloney murine leukemia virus reverse transcriptase (Toyobo, Osaka, Japan). From these RT products, cDNA fragments for SgIGSF and glyceraldehyde 3-phosphate dehydrogenase (GAPDH), 300 bp and 150 bp in size, respectively, were amplified. The sequences of the primers used were as follows: SgIGSF forward primer, 5'-TTGCTCAT- 
CATTCTGGGCCG-3'; SgIGSF reverse primer, 5'-CAGTCTCGCATCTCTCCACC-3'; GAPDH forward primer, 5'GATGGGTGTGAACCACGAGA-3'; GAPDH reverse primer, 5'-ATGGACTGTGGTCATGAGCC-3'. Conventional RTPCR was first performed for 30 cycles using TaqDNA polymerase (ExTaq; Takara Biomedicals, Kusatsu, Japan) in a DNA thermal cycler (MJ Research, Watertown, MA), and the amplified products were analyzed with agarose-gel electrophoresis. For quantitative realtime RT-PCR, a mixture containing the RT product, the primers for SgIGSF or GAPDH, and LightCycler DNA Master SYBR Green I (Roche Diagnostics, Indianapolis, IN) were first incubated at $95^{\circ} \mathrm{C}$ for $10 \mathrm{~min}$, followed by 50 cycles of incubation at $95^{\circ} \mathrm{C}$ for $10 \mathrm{sec}, 55^{\circ} \mathrm{C}$ for $5 \mathrm{sec}$, and $72^{\circ} \mathrm{C}$ for $15 \mathrm{sec}$, using a LightCycler System 305S (Roche Diagnostics). To generate the standard curves, serial dilutions of purified cDNA fragments for SgIGSF and GAPDH were amplified with their respective primers. The relative level of SgIGSF mRNA in each experimental sample compared with the control sample was calculated by applying the $\mathrm{Ct}$ value to the standard curve, and was normalized with the relative level of GAPDH mRNA obtained in the same way. Values for 6 samples for each experimental condition ( 2 samples $\times 3$ experiments) were expressed as the mean \pm standard deviation (SD). Statistical difference among multiple values was analyzed by one-factor analysis of variance (ANOVA), followed by comparison between two values by Bonferroni's post-hoc test. A P value less than 0.05 was considered significant.

\section{Western blotting}

Rabbit polyclonal IgG antibody against the synthetic peptide spanning the carboxyl terminal 15-amino acids for mouse SgIGSF was generated as described previously [24]. Frozen mouse olfactory mucosa were homogenized in a lysis buffer composed of $1 \%$ Nonidet $\mathrm{P} 40,0.5 \%$ sodium deoxycholate, $50 \mathrm{mM}$ Tris- $\mathrm{HCl}, \mathrm{pH} 7.5,150 \mathrm{mM} \mathrm{NaCl}$, and proteinase inhibitor cocktail (Roche, Mannheim, Germany). After centrifugation at $3000 \mathrm{rpm}$, the supernatants were examined for protein concentration using a BCA protein assay kit (Pierce, Rockford, IL) and used as cell lysates. Aliquots of cell lysates at $20 \mu \mathrm{g}$ protein/lane were separated by electrophoresis in a $15 \%$ polyacrylamide gel in the presence of $0.1 \%$ SDS and then transferred to a PVDF membrane (BioRad Laboratories, Hercules, CA). After treatment with 5\% non-fat skim milk in PBS, the membrane was incubated with rabbit anti-SgIGSF antibody at a concentration of 0.05 $\mu \mathrm{g} / \mathrm{ml}$ or rabbit anti- $\alpha$-tubulin antibody (Sigma Aldrich, Co., St. Louis, MO) at 1:10,000 dilution for $2 \mathrm{hr}$ at room temperature. After a wash with PBS plus $0.05 \%$ Tween 20 , the membrane was incubated with horseradish peroxidaseconjugated anti-rabbit IgG antibody (Dako, Glostrup, Denmark) at 1:2000 dilutions for $1 \mathrm{hr}$. Immunoreaction was detected with X-ray film (Kodak X-OMAT AR) after treatment of the membrane with the chemiluminescence kit ECLplus (Amersham Pharmacia Biotech, Uppsala, Sweden). Developed X-ray film was converted to an image file by scanning with an Epson GT-9800F scanner and the optical density of the immunoreactive band was quantitated with an Image Gauge version 3.41 software (Fuji Photo Film, Co., Tokyo, Japan). The relative level of SgIGSF in each experimental sample compared with the control sample was calculated in terms of the optical density of the immunoreactive band, and was normalized with the relative level of $\alpha$-tubulin obtained in the same way. Values for 3 samples (1 sample each from 3 experiments) in each experimental condition were expressed as the mean \pm SD. Statistical difference among multiple values was analyzed by one-factor ANOVA, followed by comparison between two values by Bonferroni's post-hoc test. A P value less than 0.05 was considered significant.

\section{Immunohistochemistry}

Decalcified mouse heads in paraffin blocks were cut in 5 - $\mu \mathrm{m}$-thick frontal sections using a microtome and mounted on silane-coated glass slides. After deparaffinization in xylene, the sections were subjected to antigen-retrieval by heating at $100^{\circ} \mathrm{C}$ for $5 \mathrm{~min}$ in $10 \mathrm{mM}$ citrate buffer $(\mathrm{pH}$ 6.0) using microwave and allowing them to slowly cool down to room temperature. Thereafter, the sections were treated with $0.3 \% \mathrm{H}_{2} \mathrm{O}_{2}$ in methanol for $10 \mathrm{~min}$ to inhibit intrinsic peroxidase activity, and 10\% normal goat serum (Dako, Glostrup, Denmark) for $1 \mathrm{hr}$ to prevent nonspecific antibody binding. For immunohistochemistry by enzyme-detection method, these pretreated sections were incubated overnight at room temperature with rabbit anti-SgIGSF antibody at a concentration of $1 \mu \mathrm{g} / \mathrm{ml}$ in PBS. To confirm the specificity of immunoreaction, the antibody was absorbed by incubating with $10 \mu \mathrm{g} / \mathrm{ml}$ of the peptide antigen for SgIGSF for $1 \mathrm{hr}$ at $4^{\circ} \mathrm{C}$ prior to use. After washing, the site of immunoreaction was visualized by incubating the sections successively with biotinylated anti-rabbit IgG antibody (Vector Laboratories, Burlingame, CA) at 1:200 dilution for $1 \mathrm{hr}$, horse radish peroxidase-conjugated streptavidin (Dako) at 1:300 dilution for $1 \mathrm{hr}$, and $0.01 \%$ diaminobenzidine tetrahydrochloride in the presence of $0.02 \% \mathrm{H}_{2} \mathrm{O}_{2}$ in $50 \mathrm{mM}$ Tris- $\mathrm{HCl}, \mathrm{pH} 7.5$ for about $10 \mathrm{~min}$. The sections were subjected to observation under an Olympus BX50 microscope.

For fluorescent double-immunostaining, the pretreated sections were incubated with a mixture of rabbit antiSgIGSF antibody $(1 \mu \mathrm{g} / \mathrm{ml})$ and mouse anti-proliferating cell nuclear antigen (PCNA) antibody (1:40 dilution, Dako) or mouse anti-Gap43 antibody (1:50 dilution, Chemicon, Temecula, CA) overnight at $4^{\circ} \mathrm{C}$. After washing in PBS, the sections were incubated with a mixture of FITC-labeled anti-rabbit IgG (1:50 dilution, Rockland, Philadelphia, PA) and Cy3-labeled anti-mouse IgG (1:100 dilution, Chemicon) for $1 \mathrm{hr}$ at room temperature. They were then mounted in glycerol containing $1 \mathrm{mg} / \mathrm{ml}$ p-phenylenediamine and subjected to observation with an Olympus BX50/BX-FLA fluorescent microscope.

For electron-microscopic immunocytochemistry, the pre-embedding immunoreaction method was adopted. Frozen specimens were cut in $15 \mu$ m-thick sections using a 
cryostat and mounted on plastic slides. After immunostaining with anti-SgIGSF antibody in the same way as for light microscopy, the sections were postfixed in $0.5 \%$ $\mathrm{OsO}_{4}$ for $30 \mathrm{~min}$, stained with $1 \%$ uranyl acetate for 20 min, dehydrated in graded ethanol series, and embedded in Glicidether 100 (Selva Feinbiochemica, Heidelberg, Germany). Ultrathin sections were cut with an ultramicrotome and subjected to observation with a JEM-1210 electron microscope (JEOL, Tokyo, Japan).

\section{Results}

\section{Morphology of the olfactory epithelium before and after olfactory nerve transection}

Olfactory epithelia at various times after transection of the olfactory nerves were examined with hematoxylin-eosin staining (Fig. 1). Before transection, the nuclei of sustentacular cells, olfactory cells, and basal cells were arranged in order from the apical surface to the basement membrane (Fig. 1A). By 4 days after transection, the epithelium reduced its thickness substantially, the olfactory cilia on the apical surface disappeared, and the nuclei of cells in the middle portions of the epithelium disappeared. At 7 to 15 days after transection, the epithelium regained its thickness gradually with increased number of cells in the middle portions of the epithelium. By 35 days after transection, the thickness and cellular composition of the epithelium, as well as the olfactory cilia, completely restored their states to the levels they maintained before transection. These observations were consistent with the changes in the olfactory epithelium after olfactory nerve transection described in the literature $[7,30]$. As shown in Figure 1B, these changes may be interpreted in terms of cellular composition as follows: mature olfactory cells undergo apoptosis and disappear by 4 days; basal cells proliferate, migrate, and differentiate into immature olfactory cells around 4-7 days; immature olfactory cells extend their dendrites and axons around 11-15 days; and mature olfactory cells are formed by 35 days after transection.

\section{Expression of SgIGSF mRNA after olfactory nerve transection}

Expression of SgIGSF mRNA in the olfactory mucosa at various times after transection of olfactory nerves was examined with RT-PCR analyses (Fig. 2). In conventional RTPCR, a single band for amplified SgIGSF cDNA was obtained in all total RNA samples (Fig. 2A). Samples at 7 days after the transection tended to show higher levels of mRNA expression than other samples. Therefore, the samples were further examined with real time quantitative RT-PCR (Fig. 2B). Compared with the control level before transection, the level of SgIGSF mRNA was not significantly changed after 4 days, but showed a substantial increase after 7 days, reaching levels 4.1-times higher than that of the control $(\mathrm{P}<0.05)$. After 11 and 15 days, the levels were decreased to about half of the peak at 7 days but still were significantly higher than the control $(\mathrm{P}<0.05)$. By 35 days after transection, the

A
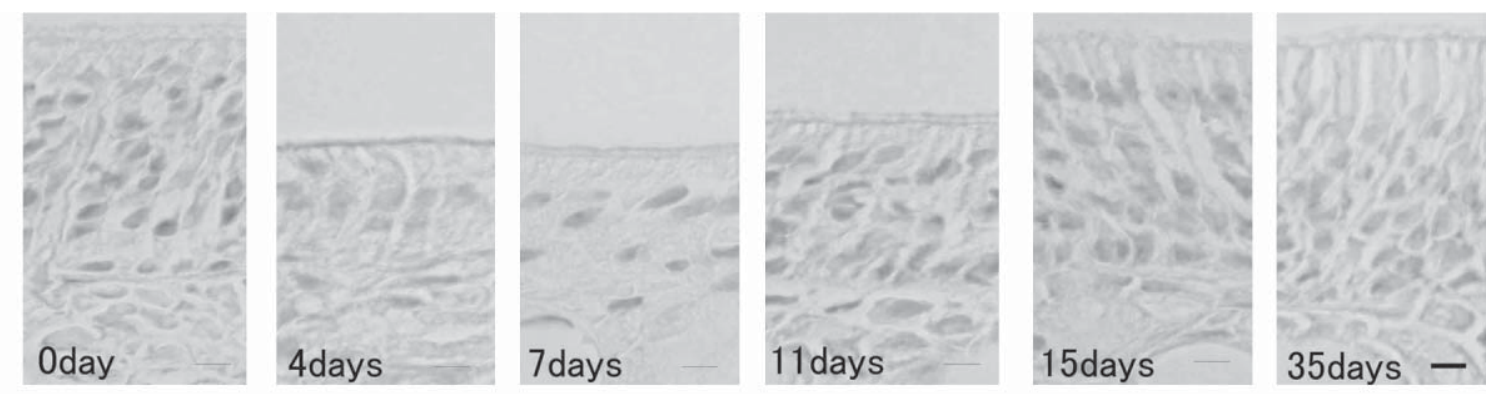

B

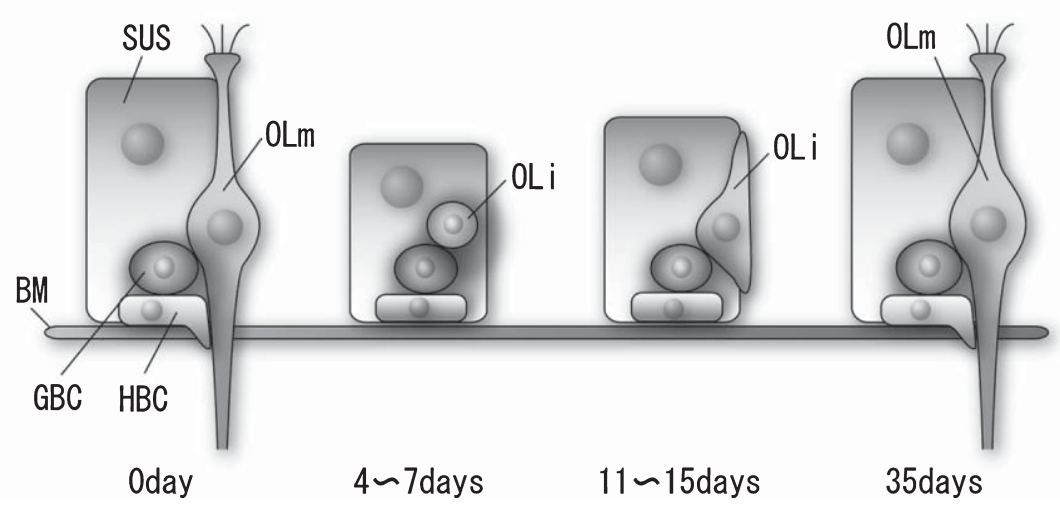

Fig. 1. Regeneration of olfactory epithelium after olfactory nerve transection. A: hematoxylin-stained olfactory epithelia from mice 0 day (control) and 4, 7, 11, 15 and 35 days after the transection. Bar=10 $\mu \mathrm{m}$. B: Schema representing cellular composition of olfactory epithelia from mice 0, 4-7, 11-15 and 35 days after transection. BM, basement membrane; HBC, horizontal basal cell; GBC, globose basal cell; SUS, sustentacular cell; OLm, mature olfactory cell; OLi, immature olfactory cell. 
A

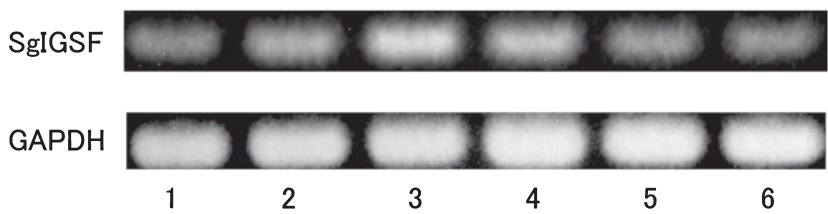

B

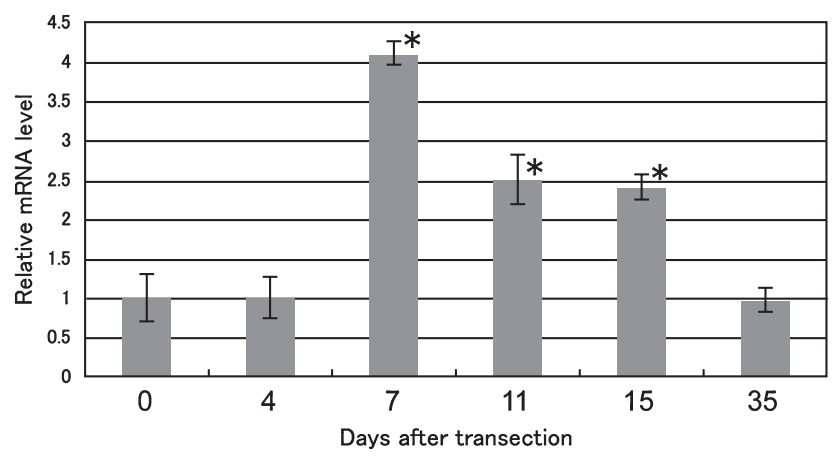

Fig. 2. Conventional (A) and real-time quantitative (B) RT-PCR analyses for SgIGSF mRNA expression in olfactory mucosa after olfactory nerve transection. (A) Amplified products for total RNA from olfactory mucosa $0,4,7,11,15$ and 35 days after transection Y (lanes 1-6) were electrophoresed and stained with ethidium bromide. A representative result is shown. (B) The relative level of SgIGSF mRNA against GAPDH mRNA at 0 day, arbitrarily set as 1 , is plotted against the corresponding values after transection. Each point represents mean \pm SD of 6 samples. * Significantly different from 0 day value $(\mathrm{P}<0.05)$.

SgIGSF mRNA declined to a level not significantly different from that of the control.

\section{Expression of SgIGSF protein after olfactory nerve transection}

Expression of SgIGSF protein in the olfactory mucosa at various times after transection of the olfactory nerves was examined with Western blot analysis (Fig. 3). The cerebrum of the control mouse showed multiple immunopositive bands ranging from $100 \mathrm{kDa}$ to $45 \mathrm{kDa}$ in size, whereas the lung showed a single $100 \mathrm{kDa}$ band (Fig. 3A). These results were consistent with our previous report [25]. All olfactory mucosa samples formed a single $100 \mathrm{kDa}$ band identical in size with that from the lung. Relative intensity of the immunopositive band representing the level of SgIGSF protein was unchanged from the control at 4 days after transection, but showed a significant increase after 7 days $(\mathrm{P}<0.05)$ (Fig. $3 \mathrm{~B})$. The level reached a peak at 11 days, representing 2.3times the control level $(\mathrm{P}<0.05)$, and then declined gradually to the control level by 35 days.

\section{Localization of SgIGSF protein after olfactory nerve transection}

Paraffin sections of mouse olfactory mucosa at various time points after transection of the olfactory nerves were examined for localization of SgIGSF with light microscopic
A

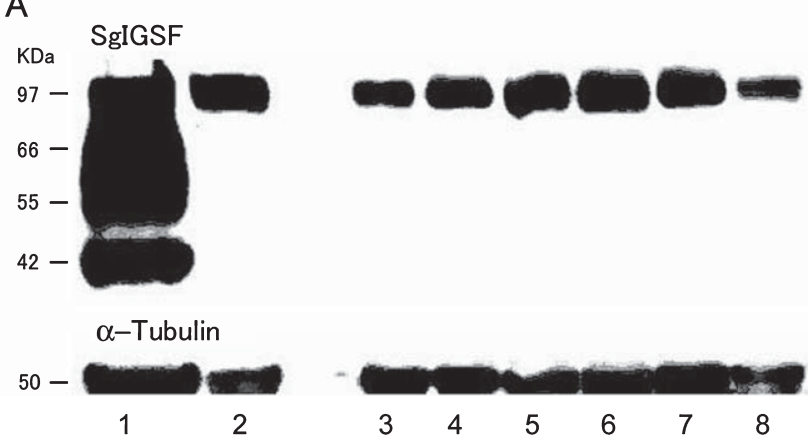

B

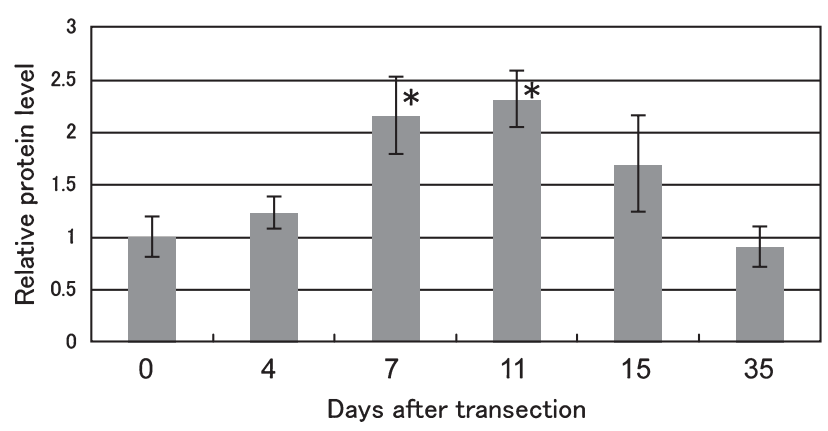

Fig. 3. Western blot analysis for SgIGSF in olfactory mucosa after olfactory nerve transection. (A) Protein samples were electrophoresed, blotted and stained with anti-SgIGSF and anti- $\alpha$-tubulin antibodies. A representative result is shown. Lanes represent cerebrum (lane 1) and lung (2) from the control mouse, and olfactory musosa from mice 0 day (3), 4 days (4), 7 days (5), 11 days (6), 15 days (7) and 35 days (8) after the transection. Molecular weights $(\mathrm{kDa})$ are indicated. (B) Relative intensity of SgIGSF band against $\alpha$-tubulin at 0 day is set as 1 and the corresponding values after transection are plotted. Each point represents mean \pm SD of 3 samples. * Significantly different from 0 day value $(\mathrm{P}<0.05)$.

immunohistochemistry (Fig. 4). In the olfactory mucosa of normal animals without nerve resection, a weak immunoreactivity was distributed all over the olfactory epithelium and also in the olfactory nerves, i.e., the axons of olfactory cells, located in the mucosa propria. Relatively strong immunoreactivity, however, was localized mainly in the apical portions of the epithelium, where the dendrites of mature olfactory cells are known to be in contact with sustentacular cells. Four days after resection, when the epithelium became much thinner due to loss of mature olfactory cells, immunoreactivity of moderate intensity remained in the apical epithelial portions, seemingly on the membrane of sustentacular cells. At 7 days, some cells strongly immunopositive for SgIGSF were observed in the middle portions of the epithelium. The immunoreactivity in these cells appeared to be localized to the cell membrane. At 11 days, the strongly immunopositive cells increased substantially in number with increase in the thickness of the epithelium. At 15 days, when the epithelium was mostly restored to its original thickness, the immunoreactivity decreased in intensity in the middle portions of the epithelium, but instead increased in its apical portions, 

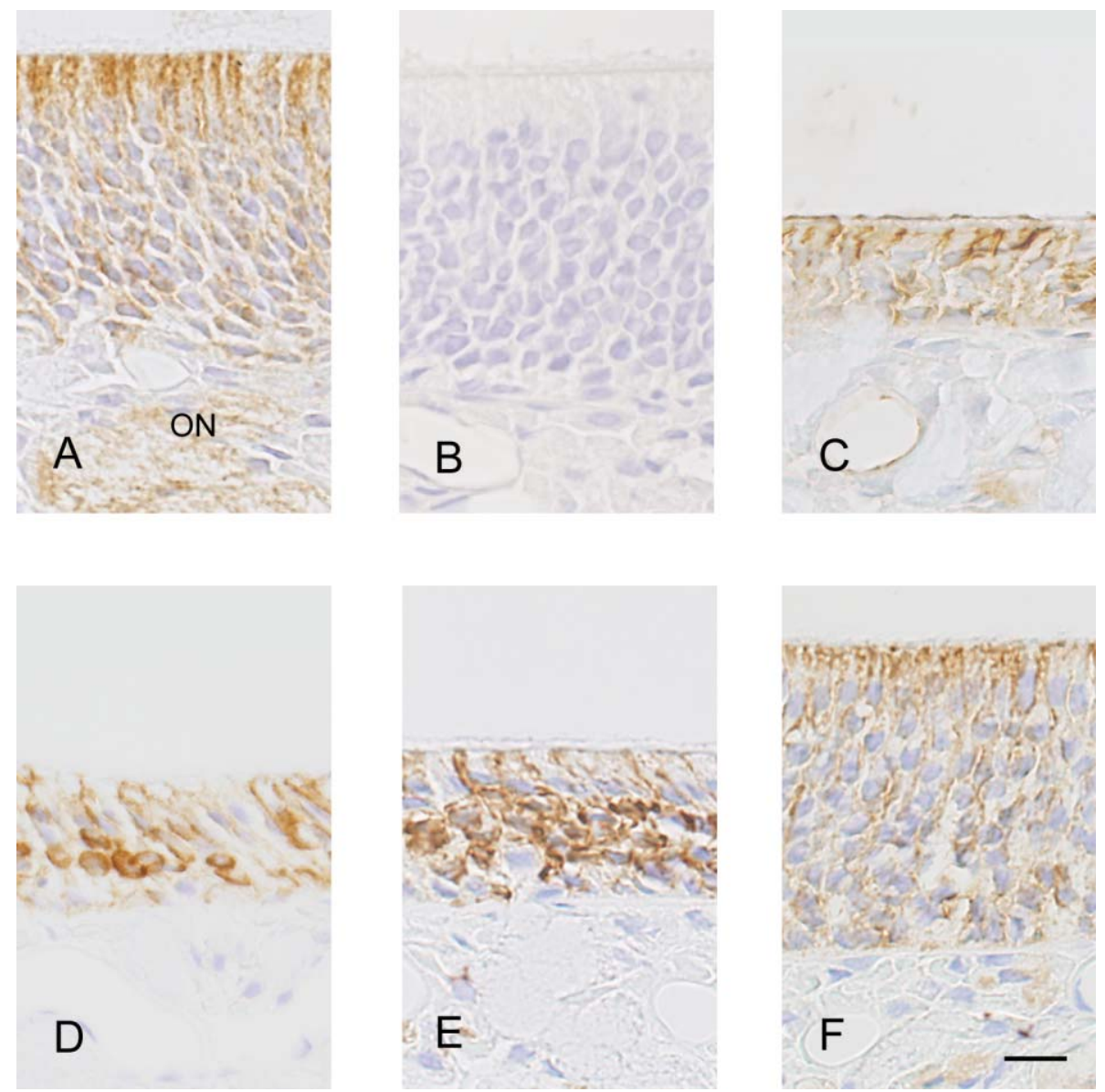

Fig. 4. Immunohistochemical localization of SgIGSF in olfactory epithelia from mice 0 day $(\mathbf{A}, \mathbf{B}), 4$ days $(\mathbf{C}), 7$ days $(\mathbf{D}), 11$ days $(\mathbf{E})$ and 15 days $(\mathbf{F})$ after olfactory nerve transection. Paraffin sections were incubated with anti-SgIGSF antibody (A, C, D, E, F) or antibody preabsorbed with peptide antigen for SgIGSF (B). ON, olfactory nerves. Bar=10 $\mu \mathrm{m}$.
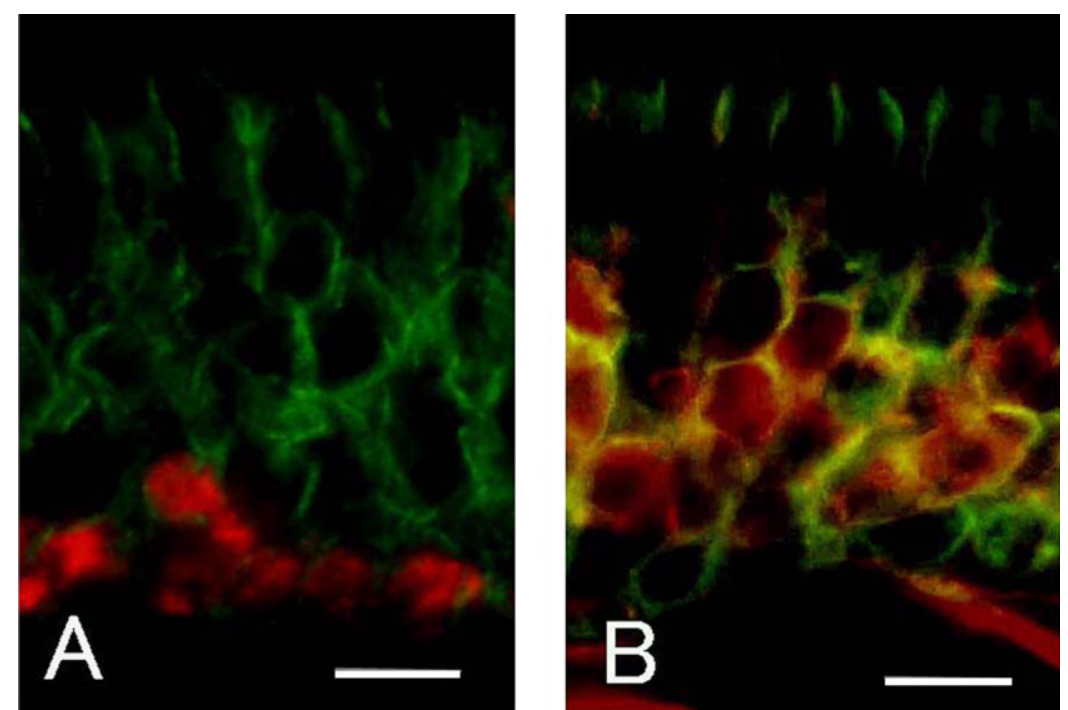

Fig. 5. Double immunostaining for SgIGSF and PCNA (A), and SgIGSF and Gap43 (B) in olfactory epithelium 11 days after olfactory nerve transection. Merged pictures for both immunofluorescence are presented. (A) Nuclear PCNA (red) and membrane SgIGSF (green) do not occur in the same cells. (B) Cytoplasmic Gap43 (red) and membrane SgIGSF (green) mostly occur in the same cells. Bars=10 $\mu \mathrm{m}$. 

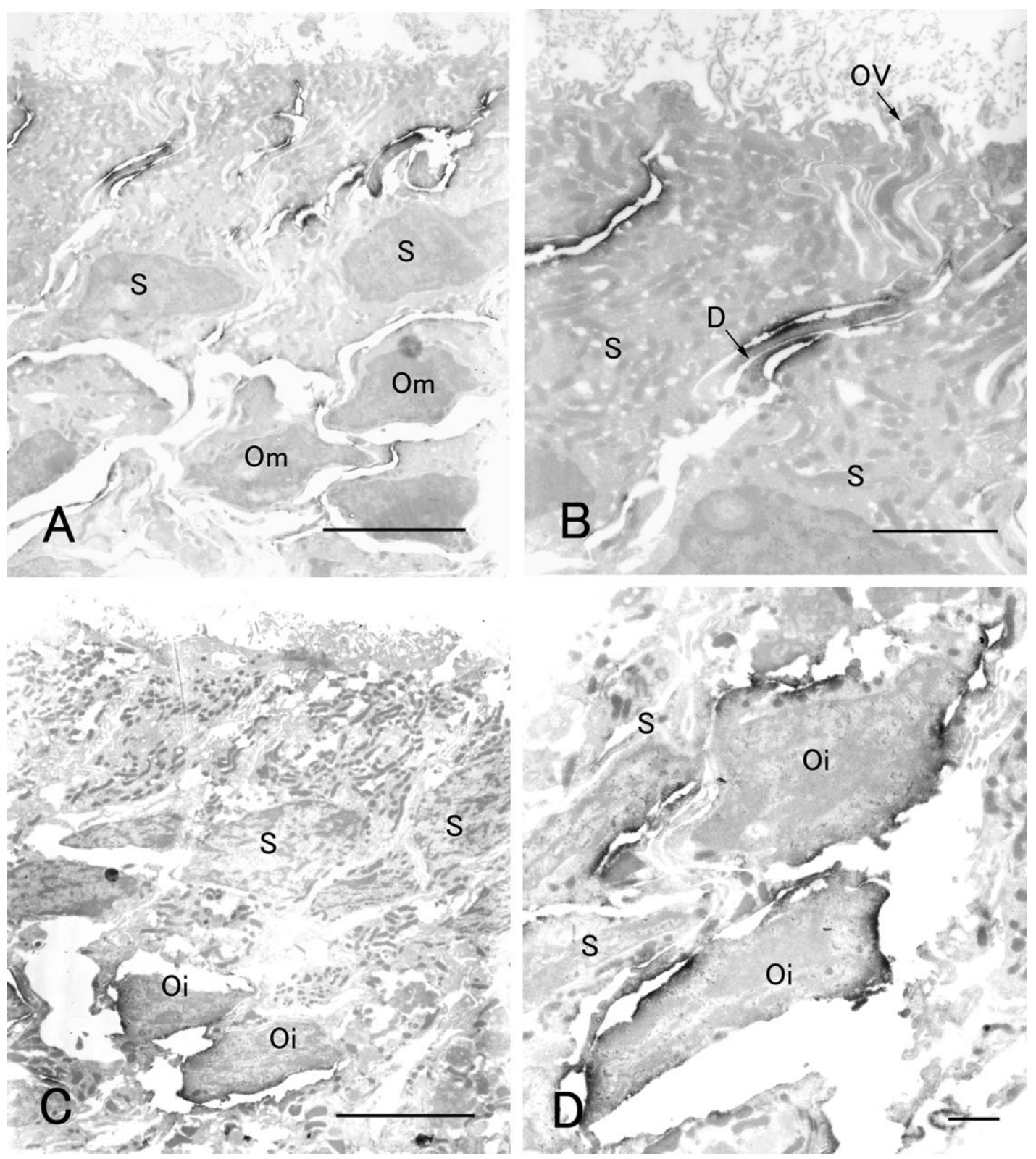

Fig. 6. Immunoelectron microscopy for SgIGSF in olfactory epithelia 0 day (A, B) and 11 days (C, D) after olfactory nerve transection. (A) Intense immunoreactivity is localized to cell surface in apical portions of the epithelium. Weaker immunoreactivity is recognized on the cell surface in other portions. S, sustentacular cells; Om, mature olfactory cells. (B) At higher magnification of apical epithelial portions, the membrane of the dendrites (D) of mature olfactory cells and the opposing membrane of sustentacular cells (S) are intensely immunostained. Membrane portions at the base of the olfactory vesicle (OV) are immunonegative. (C) Intense immunoreactivity is localized to the middle portions of the epithelium where immature olfactory cells (Oi) exist, whereas it is not recognized in the apical portions of the epithelium. S, sustentacular cells. (D) At higher magnification of middle epithelial portions, the surface of immature olfactory cells (Oi) and the opposing membrane of sustentacular cells (S) are intensely immunostained. Bars $=5 \mu \mathrm{m}(\mathbf{A}, \mathbf{C})$ and $1 \mu \mathrm{m}(\mathbf{B}, \mathbf{D})$.

exhibiting a pattern similar to the one before olfactory nerve transection. The negative control sections incubated with the antibody solution preabsorbed with the peptide antigen showed no immunostaining in any portions.

To identify the cells with strong SgIGSF immunoreactivity observed at 7 to 11 days after olfactory nerve transection, we performed double immunostaining in 11-day specimens for SgIGSF and PCNA, the marker of proliferating cells, or Gap43, the marker of immature olfactory cells (Fig. 5). Cells with nuclear staining for PCNA, located close to the basement membrane, had no intense membrane staining for SgIGSF. In contrast, cells with cytoplasmic staining for Gap43, located in the middle portions of the epithelium, were mostly immunopositive for SgIGSF. These results suggested that the immature olfactory cells occurring 7-11 days after the transection express SgIGSF at higher levels. 
Finally, we confirmed the cellular and subcellular localization of SgIGSF in the olfactory epithelium with immunoelectron microscopy (Fig. 6). In the epithelium of the control animal before transection, weak immunoreactivity for SgIGSF was distributed on the membrane of all mature olfactory, sustentacular, and basal cells, whereas intense immunoreactivity was restricted to the opposing membrane portions of olfactory cell dendrites and sustentacular cells (Fig. 6A, B). The base of the olfactory vesicle, where the junctional complexes exist, did not show intense immunoreactivity. At 11 days after transection, intense immunoreactivity was located on the membrane of immature olfactory cells, which were characterized by their location in the middle portions of the epithelium and incomplete extension of dendrites and axons (Fig. 6C, D).

\section{Discussion}

The present study has demonstrated for the first time the expression and localization of the cell adhesion molecule SgIGSF in the olfactory mucosa in normal adult mice. Immunohistochemistry indicates that SgIGSF is distributed on the membrane of all cell populations in the olfactory epithelium, with special enrichment in the apical portions of the epithelium where the dendrites of olfactory cells are in contact with sustentacular cells. On Western blotting, SgIGSF in the olfactory mucosa has a single band with a molecular weight of approximately $100 \mathrm{kDa}$. Past studies reported that SgIGSF showed multiple bands of $45 \mathrm{kDa}$ to $100 \mathrm{kDa}$ in the cerebrum, a single band of $45 \mathrm{kDa}$ in the sciatic nerve, a single strong band of $120 \mathrm{kDa}$ and weaker bands of $90 \mathrm{kDa}$ and $100 \mathrm{kDa}$ in the testis, and a single band of $100 \mathrm{kDa}$ in the liver and lung [24, 25]. Such differences in the molecular weight of SgIGSF were ascribed to the extent of glycosylation of the protein molecule. The present results have revealed that the molecular weight of SgIGSF in the olfactory mucosa is different from that in the nervous system, but the same as that in the epithelial organs represented by the lung. This is interesting in view of the fact that olfactory cells belong to the neurons and at the same time the epithelial cells undergoing continuous turnover.

SgIGSF belongs to the subclass of IGSF adhesion molecules known as the nectins and nectin-like molecules (necls) [19]. Members of these subclasses are composed of three extracellular Ig-like domains, a single transmembrane domain and a cytoplasmic domain. Through interaction of their Ig-like domains, these adhesion molecules can form cis-dimers on the same cell membrane and trans-dimers on the opposing cell membranes. Homophilic binding between SgIGSF molecules on opposing cell membranes usually means trans-dimer formation between the cis-dimers of SgIGSF on both sides $[12,18]$. An in vitro study showing that cultured non-neural cells forced to express SgIGSF form synapses with cultured neural cells, indicates a homophilic binding between SgIGSF molecules and suggests that SgIGSF may play biological roles in the nervous system [2]. However, it is unknown if the homophilic binding can solely account for all SgIGSF-mediated neuron-neuron and neuron-glia interactions in vivo in the nervous system. On the other hand, in the cases of the interactions between mast cells and fibroblasts and between spermatogenic and Sertoli cells, the latter cell populations do not express SgIGSF, implying involvement of a heterophilic binding between SgIGSF and other adhesion molecules [9, 24].

In the present results, SgIGSF was detected by Western blotting and immunohistochemistry in the olfactory epithelium 4 days after nerve resection, when mature olfactory cells have degenerated. Together with the results of immunoelectron microscopy showing SgIGSF immunoreactivity on the opposing membrane portions of olfactory and sustentacular cells, it is suggested that the homophilic binding between SgIGSF molecules is primarily involved in the interaction among cells in the olfactory epithelium. The enrichment of SgIGSF in the apical portions of the epithelium may be related to the intimate contact between adjacent sustentacular cells and between sustentacular cells and the dendrites of olfactory cells in these portions. However, it is notable that the localization of SgIGSF appears to have no relation to the junctional complex known to be formed at the base of the olfactory vesicle [13].

The present study has revealed that, during the course of degeneration and regeneration of the olfactory epithelium following olfactory nerve transection, there is a transient increase in the expression of SgIGSF mRNA and protein. This increase seems to be accounted for by abundant expression of SgIGSF in immature olfactory cells, which are derived from basal cells and on the way to differentiation into mature olfactory cells. The level of SgIGSF mRNA peaks at 7 days after transection, whereas that of SgIGSF protein peaks at 11 days. This difference may be interpreted by the differences between the life spans of mRNA and protein, taking into account the fact that SgIGSF is a membrane protein.

Basal cells in the olfactory epithelium is composed of two populations, i.e., the horizontal basal cells on the basement membrane and the globose basal cells above it. Globose basal cells are proliferative cells that are considered to contain the stem cells for all cell populations in the olfactory epithelium, as well as the precursors for olfactory cells [8]. In both the physiological turnover of olfactory cells and the regeneration of olfactory cells after olfactory nerve transection, globose basal cells may proliferate as neural precursors and differentiate into immature olfactory cells, which then migrate upward, extend dendrites and axons, and finally become mature olfactory cells $[1,17]$. Of the cell markers used in the present study, PCNA is a nuclear protein functioning in the cell cycle and mainly marks globose basal cells in the olfactory epithelium [14]. In contrast, Gap43 is a cytoplasmic signaling molecule activated by protein kinase $\mathrm{C}$ and considered to play a significant role in axonal extension. In the olfactory epithelium, Gap43 mainly marks immature olfactory cells [22]. The present study has revealed that cells with intense SgIGSF immunoreactivity, which appear in the middle portions of the olfactory epithelium 7-11 days after olfactory nerve transection, are mostly immunostained posi- 
tively for Gap43 but negatively for PCNA. These results suggest that SgIGSF expression is upregulated in immature olfactory cells that have undergone differentiation from neural precursor cells.

In regard to the biological roles of SgIGSF, Takeuchi et al. and Takai et al., based on in vitro studies using cultured cells, have proposed a model described as follows. SgIGSF (Necl-2) monomers expressed on opposing cell membranes first form a homophilic trans-dimer through interaction of their Ig-like domains, thereby causing the cells to be attached temporarily. A series of events follows, involving cis-dimer formation between SgIGSF and Nectin-3, trans-dimer formation between SgIGSF and Nectin-3, and replacement of SgIGSF with Nectin-1 or 2, makes the cell adhesion more stable $[18,19]$. The present results have raised the possibility that SgIGSF plays important roles in the temporary cell adhesion that occur in the process of migration of immature olfactory cells among sustentacular cells and extension of dendrites and axons by immature olfactory cells to become mature olfactory cells.

Finally, there may be an intracellular signaling mechanism by which cell adhesion through SgIGSF causes cellular responses in terms of cytoskelton movement and/or gene expression. In the case of E-cadherin-mediated cell adhesion found in the adherens junction of epithelial cells, homophilic binding between cadherin molecules activates intracellular signaling mechanisms through the cytoplasmic proteins $\alpha$ and $\beta$-catenins, which function as adapters between the cytoplasmic domain of cadherins and actin filaments [11]. Also, the cytoplasmic domain of nectins binds to afadin that is associated with actin filaments [19]. In the cultured cell systems, the cytoplasmic domain of SgIGSF is known to have two motifs that bind with several cytoplasmic proteins, namely, Dal1/Protein 4.1B, CASK, and syntenin [2, 27]. Whether these potential signaling molecules are involved in the regeneration of olfactory cells is unknown and warrants further investigation.

\section{Acknowledgment}

This work was supported in part by a research grant from the Honjin Foundation in Kanazawa University to TW.

\section{References}

1. Beites, C. L., Kawauchi, S., Crocker, C. E. and Calof, A. L. (2005) Identification and molecular regulation of neural stem cells in the olfactory epithelium. Exp. Cell Res. 306; 309-316.

2. Biederer, T., Sara, Y., Mozhayeva, M., Atasoy, D., Liu, X., Kavalali, E. T. and Sudhof, T. C. (2002) SynCAM, a synaptic adhesion molecule that drives synapse assembly. Science 297; $1525-1531$.

3. Brummendorf, T. and Rathjen, F. G. (1995) Cell adhesion molecules 1: immunoglobulin superfamily. Protein Profile 2; 9631108.

4. Fujita, E., Urase, K., Soyama, A., Kouroku, Y. and Momoi, T. (2005) Distribution of RA175/TSLC1/SynCAM, a member of the immunoglobulin superfamily, in the developing nervous system. Brain Res. Dev. Brain Res. 154; 199-209.
5. Gomyo, H., Arai, Y., Tanigami, A., Murakami, Y., Hattori, M., Hosoda, F., Arai, K., Aikawa, Y., Tsuda, H., Hirohashi, S., Asakawa, S., Shimizu, N., Soeda, E., Sakaki, Y. and Ohki, M. (1999) A 2-Mb sequence-ready contig map and a novel immunoglobulin superfamily gene IGSF4 in the LOH region of chromosome 11q23.2. Genomics 62; 139-146.

6. Graziadei, P. P. (1973) The ultrastructure of vertebrate olfactory mucosa. In "The Ultrastructure of Sensory Organs", ed. by I. Friedmann, North-Holland \& American Elsevier, Amsterdam \& New York, pp. 267-305.

7. Graziadei, G. A. and Graziadei, P. P. (1979) Neurogenesis and neuron regeneration in the olfactory system of mammals. II. Degeneration and reconstitution of the olfactory sensory neurons after axotomy. J. Neurocytol. 8; 197-213.

8. Huard, J. M., Youngentob, S. L., Goldstein, B. J., Luskin, M. B. and Schwob, J. E. (1998) Adult olfactory epithelium contains multipotent progenitors that give rise to neurons and non-neural cells. J. Comp. Neurol. 400; 469-486.

9. Ito, A., Jippo, T., Wakayama, T., Morii, E., Koma, Y., Onda, H., Nojima, H., Iseki, S. and Kitamura, Y. (2003) SgIGSF: a new mast-cell adhesion molecule used for attachment to fibroblasts and transcriptionally regulated by MITF. Blood 101; 2601-2608.

10. Kuramochi, M., Fukuhara, H., Nobukuni, T., Kanbe, T., Maruyama, T., Ghosh, H. P., Pletcher, M., Isomura, M., Onizuka, M., Kitamura, T., Sekiya, T., Reeves, R. H. and Murakami, Y. (2001) TSLC1 is a tumor-suppressor gene in human non-smallcell lung cancer. Nat. Genet. 27; 427-430.

11. Logan, C. Y. and Nusse, R. (2004) The Wnt signaling pathway in development and disease. Annu. Rev. Cell Dev. Biol. 20; 781-810.

12. Masuda, M., Yageta, M., Fukuhara, H., Kuramochi, M., Maruyama, T., Nomoto, A. and Murakami, Y. (2002) The tumor suppressor protein TSLC1 is involved in cell-cell adhesion. J. Biol. Chem. 277; 31014-31019.

13. Miragall, F., Krause, D., de Vries, U. and Dermietzel, R. (1994) Expression of the tight junction protein ZO-1 in the olfactory system: presence of ZO-1 on olfactory sensory neurons and glial cells. J. Comp. Neurol. 341; 433-481.

14. Ohta, Y. and Ichimura, K. (2000) Proliferation markers, proliferating cell nuclear antigen, Ki67, 5-bromo-2'-deoxyuridine, and cyclin D1 in mouse olfactory epithelium. Ann. Otol. Rhinol. Laryngol. 109; 1046-1048.

15. Ronn, L. C., Hartz, B. P. and Bock, E. (1998) The neural cell adhesion molecule (NCAM) in development and plasticity of the nervous system. Exp. Gerontol. 33; 853-864.

16. Rougon, G. and Hobert, O. (2003) New insights into the diversity and function of neuronal immunoglobulin superfamily molecules. Annu. Rev. Neurosci. 26; 207-238.

17. Schwob, J. E. (2002) Neural regeneration and the peripheral olfactory system. Anat. Rec. 269; 33-49.

18. Shingai, T., Ikeda, W., Kakunaga, S., Morimoto, K., Takekuni, K., Itoh, S., Satoh, K., Takeuchi, M., Imai, T., Monden, M. and Takai, Y. (2003) Implications of nectin-like molecule-2/IGSF4/ RA175/SgIGSF/TSLC1/SynCAM1 in cell-cell adhesion and transmembrane protein localization in epithelial cells. J. Biol. Chem. 278; 35421-35427.

19. Takai, Y., Irie, K., Shimizu, K., Sakisaka, T. and Ikeda, W. (2003) Nectins and nectin-like molecules: roles in cell adhesion, migration, and polarization. Cancer Sci. 94; 655-667.

20. Thornton, M. R., Mantovani, C., Birchall, M. A. and Terenghi, G. (2005) Quantification of N-CAM and N-cadherin in axotomized and crushed rat sciatic nerve. J. Anat. 206; 69-78.

21. Urase, K., Soyama, A., Fujita, E. and Momoi, T. (2001) Expression of RA175 mRNA, a new member of the immunoglobulin superfamily, in developing mouse brain. Neuroreport 121; $3217-$ 3221.

22. Verhaagen, J., Oestreicher, A. B., Gispen, W. H. and Margolis, F. L. (1989) The expression of the growth associated protein 
B50/GAP43 in the olfactory system of neonatal and adult rats. J. Neurosci. 9; 683-691.

23. Wakayama, T., Ohashi, K., Mizuno, K. and Iseki, S. (2001) Cloning and characterization of a novel mouse immunoglobulin superfamily gene expressed in early spermatogenic cells. Mol. Reprod. Dev. 60; 158-164.

24. Wakayama, T., Koami, H., Ariga, H., Kobayashi, D., Sai, Y., Tsuji, A., Yamamoto, M. and Iseki, S. (2003) Expression and functional characterization of the adhesion molecule spermatogenic immunoglobulin superfamily in the mouse testis. Biol. Reprod. 68; 1755-1763.

25. Wakayama, T., Koami, H., Yamamoto, M. and Iseki, S. (2004) Expression of the adhesion molecule spermatogenic immunoglobulin superfamily in the mouse. Acta Histochem. Cytochem. 37; 365-371.

26. Whitesides, J. G., 3rd and LaMantia, A. S. (1996) Differential adhesion and the initial assembly of the mammalian olfactory nerve. J. Comp. Neurol. 373; 240-254.

27. Yageta, M., Kuramochi, M., Masuda, M., Fukami, T., Fukuhara,
H., Maruyama, T., Shibuya, M. and Murakami, Y. (2002) Direct association of TSLC1 and DAL-1, two distinct tumor suppressor proteins in lung cancer. Cancer Res. 62; 5129-5133.

28. Yamada, D., Yoshida, M., Williams, Y. N., Fukami, T., Kikuchi, S., Masuda, M., Maruyama, T., Ohta, T., Nakae, D., Maekawa, A., Kitamura, T. and Murakami, Y. (2006) Disruption of spermatogenic cell adhesion and male infertility in mice lacking TSLC1/IGSF4, an immunoglobulin superfamily cell adhesion molecule. Mol. Cell. Biol. 26; 3610-3624.

29. Yamashita, H., Kawata, K. and Takahashi, M. (1998) Upregulation of neural growth-associated protein and neural cell adhesion molecule in mouse olfactory epithelium and axons after unilateral removal of the olfactory bulb. Eur. Arch. Otorhinolaryngol. 255; $441-445$.

30. Yee, K. K. and Rawson, N. E. (2005) Immunolocalization of retinoic acid receptors in the mammalian olfactory system and the effects of olfactory denervation on receptor distribution. Neuroscience 131; 733-743. 